\title{
El fuerte de San Lorenzo del Puntal (Cádiz) y el fuerte de San Fernando de Bocachica (Cartagena de Indias): una visión comparada
}

\author{
Gabriel Granado Castro a, Jorge Galindo Díaz ${ }^{\text {, }}$ Hugo Aragón Barreto
}

${ }^{a}$ Universidad de Sevilla, Sevilla, España, ggranado@us.es , ${ }^{b}$ Universidad Nacional de Colombia, Manizales, Colombia,jagalindod@unal.edu.co,haaragonb@unal.edu.co

\begin{abstract}
Through a comparative study of two similar fortifications in their geometric and defensive characteristics, this paper gives a description of each of them on a historical narrative that allows specifying how the processes of transformation and adaptation of buildings was given continuously, like a single project it were, away from the traditional model usually think that American fortifications were a simple adaptation of which were built in Spain .
\end{abstract}

Keywords: Cádiz, Cartagena de Indias, Ignacio Sala, fortification.

\section{Introducción}

En la ciudad española de Cádiz, puerto de embarque de muchos de los ingenieros militares que tendrían a América como destino, existe desde el siglo XVI el fuerte de San Lorenzo del Puntal, caracterizado geométricamente por tener una planta ovalada dotada de un frente curvo que mira al mar y dos semibaluartes con flanco y foso orientados hacia el frente de tierra. Su posición estratégica está claramente vinculada al fuerte de Matagorda de tal manera que entre ambos se podía producir un fuego cruzado defendiendo la entrada a la bahía.

Por su parte, en la ciudad americana de Cartagena de Indias (actual Colombia) se levantó desde mediados del siglo XVIII el fuerte de San Fernando de Bocachica, de características morfológicas similares al de Cádiz en tanto adoptó una planta de forma elíptica con un frente curvo orientado hacia el mar, que mira a la batería de San José, mientras dos baluartes dirigen sus puntas al frente de tierra.
Ambos edificios comparten no solo semejanzas geométricas y defensivas, sino también la presencia del ingeniero militar Ignacio Sala, traductor al castellano de las obras de Vauban y quien trabajaría en Cádiz entre 1730 y 1748, antes de ser enviado a América en calidad de Gobernador y Comandante General de Cartagena de Indias (Gutiérrez, 1991).

Un relato de la génesis histórica de ambos fuertes se presenta a continuación, describiendo a la vez las particularidades arquitectónicas de cada edificio con el fin de demostrar que ellas no fueron el resultado de un simple proceso de aplicación de saberes desde el centro hacia la periferia sino más bien un proceso en doble vía en el cual cada ajuste espacial o constructivo se entendía como parte de un mismo proceso de mejora continua sobre las estructuras defensivas.

\section{El fuerte de San Lorenzo del Puntal}

Situado en una de las zonas más estratégicas de la bahía de Cádiz, justo en la entrada a la misma, 
produciendo un estrechamiento del canal de acceso (Fig. 1), este lugar despertó tempranamente la necesidad de los gaditanos de fortificarlo y convertirlo en uno de los puntos claves del sistema defensivo, capaz de protegerlos de las constantes amenazas extranjeras.

Se trataba de una de las zonas de mas fácil acceso desde el mar, tal como se demostró en el fatídico ataque angloholandés de 1596.

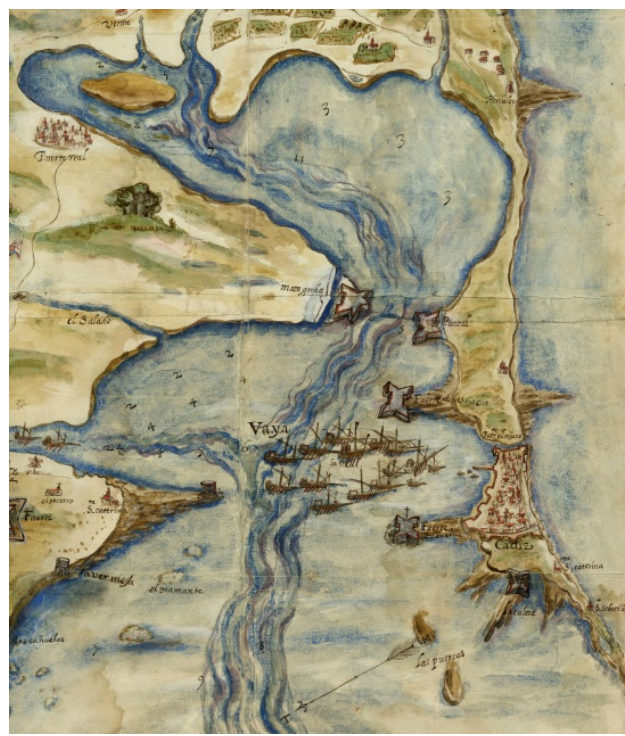

Fig. 1- Detalle de la Planta General de la ciudad de Cádiz y su bahía. Fines s. XVI. Institut Cartogràfic de Catalunya (Chías, 2011: 18).

\subsection{Orígenes}

Durante todo el siglo XVI habían sido constantes las peticiones por parte de los regentes de la ciudad respecto la necesidad de reforzar las defensas de la misma. La ciudad disponía, tan solo, de un pequeño muro de tierra y un castillo en estado ruinoso (Fernández, 1973: 3-4).

Entre estas necesidades ya se apuntaba como importante la entrada a la Bahía, con sus dos extremos: Matagorda y San Lorenzo del Puntal. A pesar de ello, los primeros esfuerzos económicos irán destinados a la defensa del territorio urbano de la propia ciudad, dejando la bahía para futuras dotaciones.
En la segunda mitad del siglo XVI empezaron a trabajar en el sistema defensivo de Cádiz diversos ingenieros italianos que introdujeron las innovaciones propias del sistema de frentes abaluartados. El primero de ellos, Giovan Battista Calvi, consideró en 1557 la necesidad perentoria de edificar tres baluartes en la parte de la ciudad que miraba hacia la bahía, si bien para los proyectados en el arrecife, en el que se incluía el fuerte del Puntal, no existía dotación económica (Martínez, 2002: 312-319). A Calvi le sucedieron Giacomo Fratino y Vespesiano Gonzaga, los cuales tampoco pudieron dar comienzo al fuerte del Puntal, empleando los escasos caudales suministrados en la defensa de la ciudad, fundamentalmente en el frente de tierra y de la bahía (Garófano, 2012: 25-27).

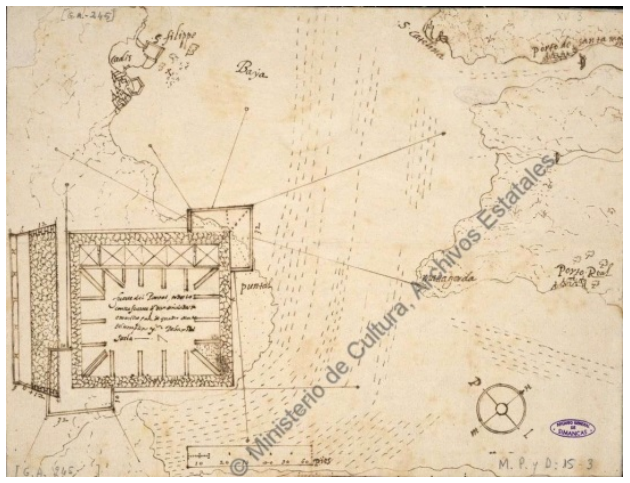

Fig. 2- Plano del Fuerte del Puntal en la bahía de Cádiz, con indicación de las obras que debían hacerse en él para su mejor defensa. 1589. (Archivo General de Simancas, Guerra y Marina, legajos 00245, 116).

No será hasta la llegada de Tiburcio Espanoqui a Cádiz en 1587 cuando se retome la posibilidad de edificar un fuerte en el sitio del Puntal. De hecho, era considerada una de las actuaciones prioritarias para el ingeniero italiano (Garófano, 2012: 28). Las obras comenzaron en 1588 y en mayo de 1589 ya estaban terminadas. Se trataba de un fuerte de pequeñas dimensiones, con escasa altura, cinco piezas de artillería y cuatro aposentos (Fig. 2). En 1594 se añadieron tres espacios más y un cuerpo de guardia (Fernández, 1973: 24). Verdaderamente no se pensaba que esta primitiva defensa pudiera cumplir su misión de impedir la entrada de naves a la bahía. 


\subsection{Su reconstrucción tras el ataque de 1596}

Las deficiencias existentes en el sistema defensivo de Cádiz se pusieron de manifiesto cuando el 30 de junio de 1596 una gran flota de buques ingleses y holandeses entró en la bahía y, sin apenas resistencia, consiguió entrar en la ciudad, arrasándola por completo (Abreu, 1866). Precisamente el desembarco de las tropas angloholandesas se realizó en las inmediaciones del fuerte del Puntal, no ofreciendo dicha pieza resistencia alguna (Concepción, 2002: 145-157) a tal punto que el fuerte fue tomado y destruido por las tropas enemigas.

Una vez la coalición angloholandesa abandonó la ciudad, la corona española estudió la recomposición de la ciudad y sus defensas. Para tal cometido Felipe II envió a Cádiz a Cristóbal de Rojas, que ya había trabajado en la ciudad como maestro de obras, pero que ahora era enviado en calidad de ingeniero. Con respecto al fuerte del Puntal se decidió deshacerlo y mejorarlo en el mismo paraje pero adelantándolo en el mar (Mariátegui, 1985: 35).

En todas las deliberaciones que se llevaron a cabo sobre la forma de fortificar la ciudad tras el saqueo de 1596, los fuertes del Puntal y Matagorda cobraban una importancia capital en el control de la bahía.

Pese a que en octubre de 1597 Felipe II decidió aprobar los proyectos de fortificación de la ciudad, entre los que se incluía la reconstrucción del fuerte de San Lorenzo del Puntal, todavía en 1608 Cristóbal de Rojas no había podido dar principio al mismo por falta de caudales y por haberse ocupado en la construcción del Castillo de Santa Catalina y la fortificación de los baluartes en el frente de tierra. Tan solo había sido posible cortar un millar de pinos para hacer estacadas para los cimientos (Fernández, 1973: 58).

En enero de 1612 informaba Cristóbal de Rojas al Rey de que la cimentación del fuerte del Puntal iba firme y muy adelantada, pero que necesitaba una mayor dotación económica para que se terminara (Mariátegui, 1985: 105).

La obra del fuerte prosiguió de manera discontinua durante los siguientes años. En
1616, muerto ya Cristóbal de Rojas, solo estaban levantados los cimientos y a finales de dicho año se realizaron reformas en el proyecto original, corrigiendo defectos que habían sido detectados en el proyecto del castillo de Santa Catalina. A partir de este momento las obras del fuerte fueron asumidas por el juez de comisión de las torres atalayas de Andalucía, Juan de la Fuente Hurtado (Fernández, 1973: 68-69).

En 1623, el fuerte se encontraba a punto de ser puesto en defensa y en el nuevo ataque angloholandés (1625), esta vez bien repelido, el fuerte se encontraba ya terminado.

El aspecto definitivo del fuerte de San Lorenzo del Puntal en el siglo XVII se puede observar en la planta del mismo que se incluye en el Atlas Heliche de mediados de dicha centuria (Fig. 3).

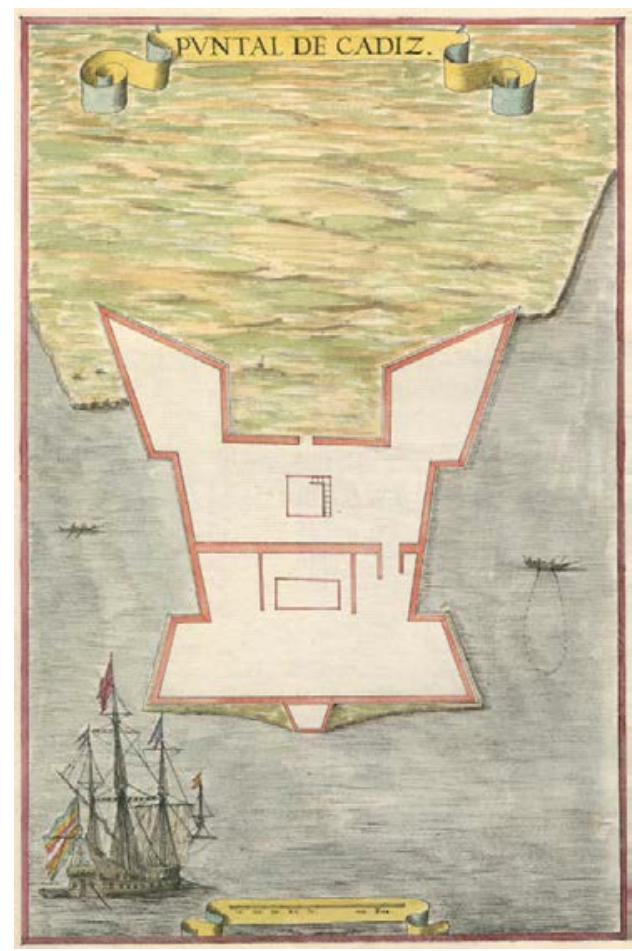

Fig. 3- Planta del Fuerte de San Lorenzo del Puntal. Mediados siglo XVII. Atlas del Marqués de Heliche (Sánchez, 2004).

\subsection{Las reformas de Ignacio Sala}

A pesar de que el fuerte requería obras para su completa terminación, tales como la finalización 
de su talud y estrada cubierta, según informe de Julio Román de Arellano de 1639 (Fernández, 1973: 84), pocas fueron las intervenciones a las que fue sometido en todo lo que restó del siglo XVII. No será hasta bien entrado el siglo XVIII, una vez creado el cuerpo de ingenieros militares tras la instauración borbónica en la monarquía, cuando se replanteó nuevamente el estado de las defensas de la ciudad de Cádiz y entre ellas, la consolidación del fuerte de San Lorenzo del Puntal.

En esa transformación de la ciudad de Cádiz como plaza fuerte en el siglo XVIII jugaría un papel crucial la figura de uno de los mejores ingenieros militares españoles de la época, Ignacio Sala, quien llegó a Cádiz en 1717 y participó casi en la totalidad de las obras que se hicieron en la ciudad hasta que en 1749 fue trasladado a Cartagena de Indias para dirigir la reconstrucción de sus defensas tras el ataque de Vernon. En los más de 30 años que Sala estuvo en Cádiz dirigió todas las obras de fortificación, permitiendo que la ciudad se convirtiera en uno de los puertos más seguros del país.

Las obras de remodelación en el fuerte de San Lorenzo del Puntal comenzaron en 1724 y se prolongaron por espacio de seis años más, modificándose su frente de tierra mediante la construcción en él de una nueva batería que si bien no llegaba a prefigurar la cortina curva que ostentará años después, sí destacaba la importancia de este flanco en su lavor defensiva de la bahía de Cádiz (Fig. 4).

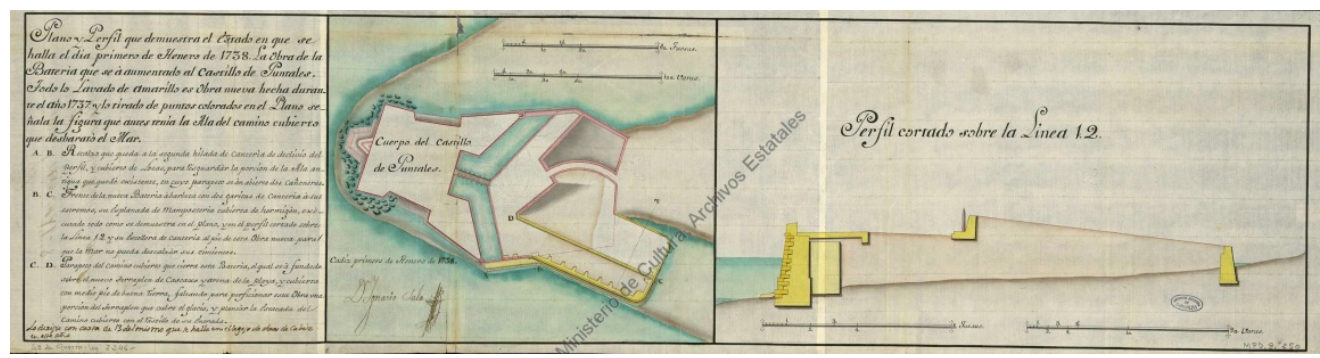

Fig. 4- Planta y perfil del castillo del Puntal. 1 de enero de 1738. Ignacio Sala (Archivo General de Simancas, Secretaría de Guerra, legajos 03246).

\section{El fuerte de San Fernando de Bocachica en Cartagena de Indias.}

En el lugar que hoy ocupa el fuerte de San Fernando, defendiendo el paso por el canal de Bocachica, sobre la isla de Tierrabomba, se levantó entre 1646 y 1649 el castillo de San Luis de Bocachica, con traza del ingeniero Juan de Somovilla y construido bajo la dirección técnica de Juan Bautista Antonelli. En 1697 el edificio fue destrozado por el ataque de Pointis y posteriormente reconstruido por Juan de Herrera y Sotomayor en 1715, aunque las obras no estaban terminadas para cuando el sitio fue atacado nuevamente, esta vez por Vernon, en 1741. Con la llegada de Ignacio Sala a Cartagena de Indias, en 1749, una de sus primeras decisiones tuvo que ver con determinar el emplazamiento y la geometría de un nuevo fuerte capaz de reemplazar al de San
Luis. Para entonces existía una propuesta formulada con anterioridad por el ingeniero Juan Bautista Mac Evan según la cual los frentes del nuevo edificio debían situarse de manera paralela al acceso para que así la batería pudiese apuntar al costado de los barcos enemigos que entraran por el canal. Contrariamente, Sala era de la idea de disponer el fuerte con su frente hacia el mar para que los barcos fuesen abatidos por la proa (Cruz, 2013).

Ambas propuestas, enfrentadas de manera radical, fueron enviadas a la Corona, en donde fue escogida la de Mac Evan, quien para entonces ya había fallecido. Su propuesta se inscribía en una planta elíptica que generaba una cortina circular enfrentada a la batería de San José (rediseñada esta sí por el propio Sala en 1751), con dos amplios baluartes que apuntaban hacia tierra firme. 


\subsection{La construcción del fuerte de San Fernando}

Los primeros trabajos de construcción del fuerte americano tuvieron lugar entre 1753 y 1760 , bajo la dirección del ingeniero militar Antonio de Arévalo. Un plano de esos años (Fig. 5) permite identificar el rápido avance de las cimentaciones y el fiel respeto por los diseños de Mac Evan.

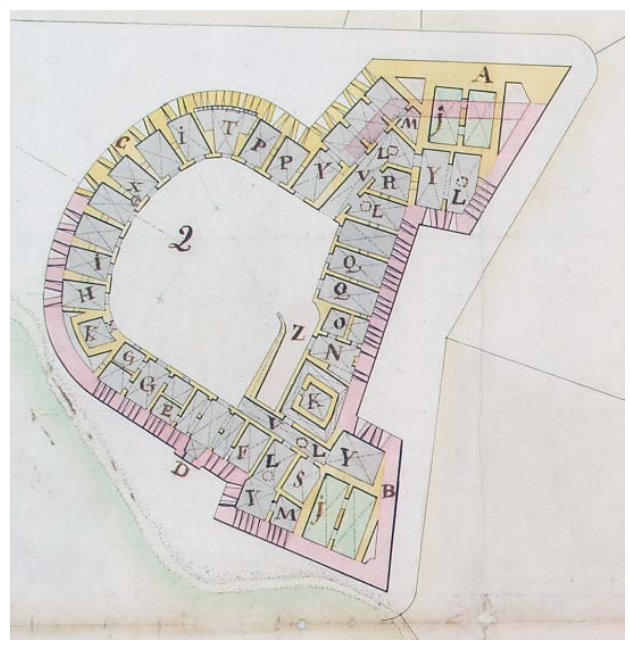

Fig. 5- Planta del fuerte de San Fernando que se esta construyendo en el canal de Bocachica, 1753, por Lorenzo de Solís. Archivo General de la Nación (Colombia), Map. 4, Ref. 402-A.

El edificio se organiza efectivamente alrededor de un gran espacio libre en forma de $\mathrm{U}$, de aproximadamente 40 por $60 \mathrm{~m}$, desde el cual se puede acceder a todos los recintos, los cuales se cubren mediante bóvedas de arista. También desde el patio se eleva una rampa de carácter monumental que conduce a una terraza almenada desde la cual se tiene una vista panorámica del entorno.

Las entradas al edificio son dos: la primera es desde el mar, de tal manera que sobre la cortina curva se levanta una imponente portada hecha en piedra dotada de un puente levadizo. La segunda entrada, más modesta, se sitúa en la parte de tierra firme a través de una pasarela que salva el foso.

Desde el punto de vista su materialidad, el edificio se resuelve a partir de potentes muros perimetrales de carga hechos con bloques de piedra caliza, a veces mezclados con ladrillos y piedras más pequeñas. Morteros elaborados a partir de cal, puzolana, polvo de ladrillo y gutagamba (resina vegetal propia del sitio), también están presentes en las mamposterías (Campos, 2003).

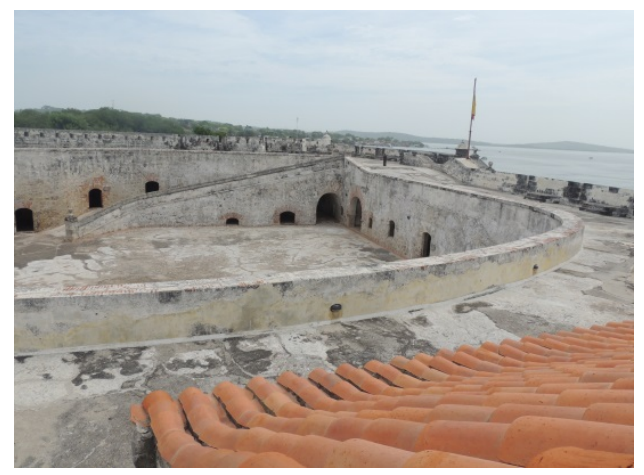

Fig. 6- Vista actual del patio y rampa del fuerte de San Fernando de Bocachica. Foto: J. Galindo.

\subsection{Cambios posteriores a su construcción}

Con posterioridad a su terminación, en 1760, el fuerte de San Fernando de Bocachica no ha sufrido alteraciones profundas en su espacialidad: el patio central, la rampa de acceso a la terraza y los salones interiores (divididos entre sí por los pesados muros de carga y pilares) se han conservado con unas pocas intervenciones de mantenimiento. Es por ello que se puede afirmar que el fuerte de Cartagena es todavía el claro resultado de más de 200 años de la experiencia de la fortificación defensiva hispanoamericana.

El fuerte de San Fernando de Bocachica nunca fue atacado y durante buena parte del siglo XIX fue olvidado y abandonado, hasta que en 1958 se empezaron a dar las primeras acciones para su recuperación.

\section{Similitudes entre los fuertes de San Lorenzo de Puntal y San Fernando de Bocachica}

Si bien hasta finales del siglo XVIII los fuertes del Puntal y San Fernando guardaban diferencias geométricas, sí tenían en su concepción varios aspectos comunes: 
(a) ambos casos eran producto de procesos de reconstrucción en donde el nuevo edificio se planteaba como una solución "mejorada" de lo existente; (b) las dos edificaciones tienen como objetivo principal la defensa del acceso a una bahía para lo cual deben dotarse de un frente de mar y reguardarse de posibles ataques que llegaran desde tierra mediante bastiones angulares que se proyectan desde el cuerpo principal separados a su vez mediante un foso de la tierra firme; sumado a lo anterior, (c) en los dos casos el fuerte está acompañado por una batería que desde el otro lado de su posición, acompaña mediante fuego cruzado su accionar ofensivo.

Adicionalmente, tanto en el fuerte de San Lorenzo de Puntal como en el de San Fernando de Bocachica se advierten similitudes de escala (Fig. 7), siendo el primero de mayor superficie y de dos pisos de altura, al menos en la actualidad.

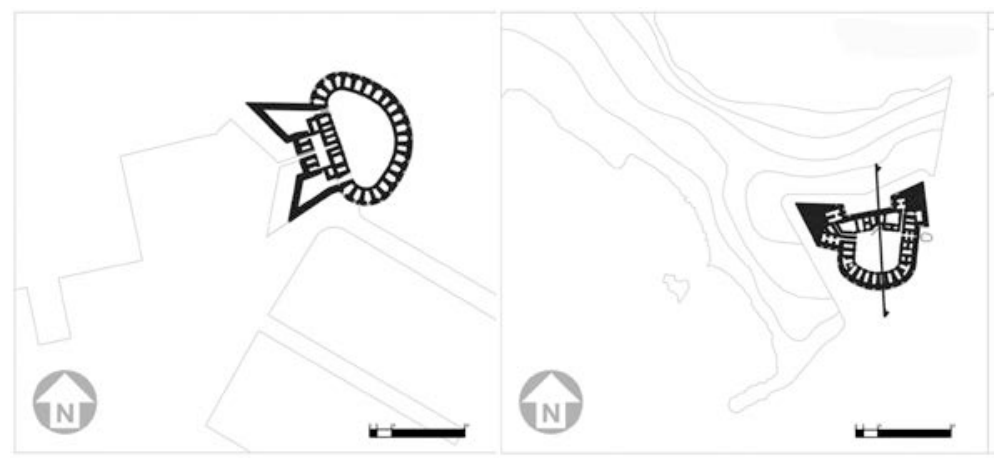

Fig. 7- Vista comparada de los fuertes de San Lorenzo de Puntal (izquierda) y San Fernando de Bocachica (derecha), dibujados a la misma escala (elaboración propia).

En cuanto al enorme parecido del trazado en planta, es importante detacar que la actual forma del fuerte de San Lorenzo del Puntal es resultado de las transformaciones acontecidas entre 1862 y 1863 con el fin de recuperar el edificio luego del considerable deterioro sufrido por el fuerte tras su decisiva participación en la defensa de la ciudad durante el asedio napoleónico (18101812). Esta remodelación cambiará completamente su estructura y geometría, siendo la misma la que goza actualmente.

Si las reformas de Sala en el siglo XVIII se centraron en el frente de tierra, esta nueva remodelación cambiará la geometría del frente de mar, utilizando, en el diseño de la planta, arcos de circunferencia (Fig. 8) y dejando obsoleta la estructura abaluartada que había defenido su forma desde su construcción en el siglo XVI.

Estas actuaciones serán realizadas por el Ingeniero Brigadier del Cuerpo de Ingenieros del
Ejército Español D. Rafael Cerero Sáenz (18341906).

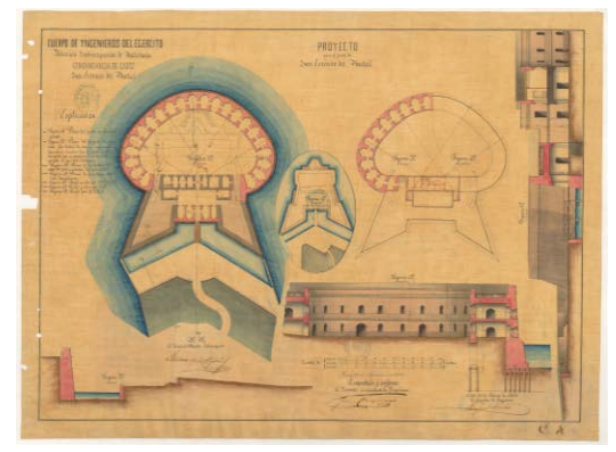

Fig. 8- Planta, perfil y vista del castillo del Puntal. 20 de febrero de 1862. Rafael Cerero (Archivo General Militar, Madrid, plano CA-1/6).

La solución adoptada (Fig. 8) emplea en el frente de mar una cortina continua formada por varios tramo circulares con diferentes centros, similar a la que se empleó en las reformas realizadas por esas fechas en el castillo de San 
Sebastián y que sin duda también fueron adoptadas a partir del diseño de San Fernando de Bocachica.

\section{Conclusiones}

El breve estudio que se reseña en esta comunicación pretende entonces demostrar la manera en que la experimentación tiplógica y constructiva era una actividad inherente a la del ingeniero militar del siglo XVIII, a través de un proceso de continuos ajustes a las estructuras fortificadas.

Los avances que se dieron en tanto en Cádiz como en otras ciudades españolas, en materia defensiva y arquitectónica, sirvieron de referente a las transformaciones aplicadas en los territorios americanos dentro de un proceso de continuidad, de tal manera que los logros allí alcanzados, también contribuían a nuevos procesos de ajuste y adaptación en la península ibérica.

En cuanto a la labor técnica y militar de Ignacio de Sala, ella está aún por ser estudiada. En el caso que nos ocupa, se advierte una coherencia en sus propuestas defensivas así como una enorme capacidad de comprender el conjunto de particularidades del territorio en el que debía adelantar sus proyectos.

Y aunque Sala no apoyara la disposición que finalmente adoptara el fuerte de San Fernando de Bocachica, se destaca su papel en la reorganización defensiva de Cartagena de Indias y su capacidad organizativa y de gestión de las obras a su cargo.

\section{Referencias}

Abreu F.P. de (1866). "Historia del Saqueo de Cádiz por los Ingleses en 1596”, en Revista Médica. Ed. Cádiz.

Cabellos, E. (1991). Cartagena de Indias: mágica acrópolis de América. Madrid: Colegio de Ingenieros de Caminos, Canales y Puertos.

Campos, D.P. (2003). Investigación del fuerte de San fernando de Bocachica: una visión integral. UPV. Valencia.

Chías P., Abad T. (dirs) (2011). El Patrimonio Fortificado. Cádiz y el Caribe: una relación transatlántica. Universidad de Alcalá. Ed. Alcalá de Hernares.

Concepción, F.J. de la (2002). Emporio de el Orbe. Tomo II. Servicio de Publicaciones de la Universidad de Cádiz. Ayuntamiento de Cádiz. Ed. Cádiz.

Cruz, P. (2013). "El ingeniero militar Ignacio Dala, Gobernador y Comandante General de Cartagena de Indias. Noticias de su pase a Indias y de su labor en las defensas de la ciudad", en: Laboratorio de Arte 25; pp. 469-481.

Fernández V. (1973). Las defensas de Cádiz en la Edad Moderna. C.S.I.C. Escuela de Estudios Hispano-Americanos. Ed. Sevilla.

Gutiérrez, R. (1991). Territorio y fortificación. Vauban, Fernández de Medrano, Ignacio Sala y Félix Prósperi. Influencia en España y América. Ed. Tuero, Madrid.

Garófano R. (2012). Cádiz Amurallada. Su registro fotográfico. Quorum Editores. Ed. Cádiz.

Martínez D. (2002). Giovan Battista Calvi. Ingeniero de las fortificaciones de Carlos V y Felipe II (1552-1565). Ministerio de Defensa. Ed. Barcelona.

Sánchez R., Testón I., Sánchez C. M. (2004). Imágenes de un imperio perdido. El Atlas del Marqués de Heliche. Plantas de diferentes Plazas de España, Italia, Flandes y Las Indias. Presidencia de la Junta de Extremadura. Ed. Badajoz. 
